\title{
Analysis of Driver Brake Operation In Near-Crash Situation Using Naturalistic Driving Data
}

\author{
Bo Cheng ${ }^{1)}$ Qingfeng Lin ${ }^{2)}$ (Tianjia Song ${ }^{3)}$ Yongwei Cui ${ }^{4)}$ Limian Wang ${ }^{5)}$ Seigo Kuzumaki ${ }^{6}$ \\ 1) Tsinghua University, State Key Laboratory of Automotive Safety and Energy \\ Beijing, 100084, China (E-mail: chengbo@tsinghua.edu.cn) \\ 2) Beihang University, School of Transportation Science and Engineering \\ Beijing, 100191, China (E-mail: linqf@buaa.edu.cn) \\ 3) • 4) • 5) Beijing Forestry University, Beijing, 100083, China \\ 6) Toyota Motor Corporation, Toyota-cho, Aichi, 471-8572, Japan
}

Received on October 8, 2009

Presented at the JSAE Annual Congress on May 21, 2010

\begin{abstract}
A large scale of field-test investigation was conducted in Beijing using Video Drive Recorder (VDR) which could record image in fron $\mathrm{t}$ of the $\mathrm{v}$ ehicle. Fifty taxis equipped with VD R were used to collect data in real traffic environments for a whole year and a larg e volume of natur alistic driving data including crashes and n ear-crashes was collected. This paper analyzed the pre-event maneuvers of drivers and studied the characteristics of braking operations of Conflict-prone drivers. Then an ev aluation method was proposed to rate a driver's accidents avoidance ability, and to evaluate the relationships between driver's brake behaviors and accident rates. The results showed that most evasive maneuvers were within $2 \mathrm{~s}$ before the most dangerous state. By comparing the near-crashes with crashes recorded by the VDR regarding traffic accidents in Beijing, great similarities were found in accident type and occurrence time between them. According to 100 rear-end near-crashes, if the braking time of drivers were delayed by $0.2 \mathrm{~s}, 17 \%$ of near-crashes would have been crashes; and if the braking forces were decreased by $0.1 \mathrm{~g}, 33 \%$ of near-crashes would have been crashes.
\end{abstract}

KEY WORDS: (Standardized) driver behavior, accident, safety, (Free) brake operation, rear-end collision, video drive recorder[C1]

\section{Introduction}

So far many study reports regarding traffic accidents have reached almost the same conclusion: driver errors before the accidents are the most important cause of traffic accidents. However, the traditional approach to analyzing accidents is usually to predict drivers' behavior before the accidents by using data gathered after the accidents occurred. Consequently, drivers' behavior prior to accidents cannot be grasped accurately and, as a result, it is difficult to describe the mechanism of the accident accurately. Thus, although many researchers used driv ing simulator to si mulate the scene of the traffic accidents and investigated the drivers' $b$ ehavior during the acciden $t$, it is questionable as to how much the experimental results can reflect the drivers' behavior prior to the actual collision.

The video drive recorder (VDR) which app eared in re cent years, can continuously record various kinds of informatio $n$ (outside traffic, vehicle status and driver behavior, etc.) before and after the accidents and thus, the appearance of this technology provided an effective method for revealing the mechan ism of traffic accidents more objectively, accurately and efficiently. VDRs were used to investigate drivers' behavior under natural driving conditions in the United States and Japan, respectively ${ }^{(1)}$ (2) (3) (4). In this study, VDRs were installed in 50 taxis in Beijing and a one-year large-scale field test was conducted, and a large volume of natur alistic driving data including crashes and nearcrashes was collected and used to study driver brake operation in emergency situations ${ }^{(5)}{ }^{(6)}$. And near-crash is defined as the circumstance that requires a rapid, evasive maneuver by Subject Vehicle (SV) to avoid a crash when a Lead Vehicle (LV) brakes suddenly. The rapid, evasive maneuver is defined as a steer ing, braking, accelerating, or any combination of control inputs that approaches the limits of the vehicle capabilities ${ }^{(1)}$.

\section{Method}

A one-year field test in Beijing was conducted using 50 taxis equipped with VDRs. The city's old urban area (within 2nd Ring Road) in Beijing is of a chessboard pattern, divided by latitudinal and longitudinal roads. In the outer city, the road pattern is circular and radial. The city's highway traffic network is comprised of five $r$ ing roads $(2 \mathrm{nd}, 3 \mathrm{rd}$, 4th, 5th and 6th Ring Roads) and 15 high-speed connecting lines. In recent years, the city, with the growth rate of 2000 vehicles and 1600 drivers per day, now has 4.4 million motor vehicles and 6 million drivers. The proportion of trips using public transport has $r$ eached to $39.3 \%$, but the proportion of trips using bicycles decreased from about $62.7 \%$ of all tr ips in 1986 to $18.1 \%$ in 2009 , and the proportion of trips using cars has reached up to $34.2 \%$. The rapid growth of $m$ otor vehicles has exacerbated the intensity of city traffic jams, and the average time of traffic congestion daily has been extended to 5 hours and the average speed of traffic flow is about $20 \mathrm{~km} / \mathrm{h}$. And meanwhile, from 2000 to 2008 , nearly 1,000 people were killed in traffic accidents per year. 
A total of 50 professional drivers including 48 males and 2 females participated in the experiment, and their age ranged from 32 to 50 years old $(43 \pm 5)$, with driving experience from 4 to 23 years $(13 \pm 4)$. The average vehicle traveled approximately 250 kilometers per day and the total amount traveled by all vehicles was about 4.5 million kilometers in this study. The VDR w as triggered if longitudinal acceleration reached $0.4 \mathrm{G}$ within $0.5 \mathrm{~s}$ or if instantaneous acceleration reaches $2 \mathrm{G}$ or above. Th e VDR could record data, such as image in front of the car, speed, acceleration(front and rear, left and right, up and down), brake signal, turning signal and GPS etc during the $18 \mathrm{~s}$ (12s before, $6 \mathrm{~s}$ after) interval before and after the near-crashes or crashes. In this study, an algorithm based on theory of image reconstruction w as developed to calculate the distance b etween SV and LV. Th e image geometry operation included the transformation of im age space coordinates and determination of pix el value. The process of camera imaging was analyzed in details and a mathematical model was developed for a bird's eye view of geometry correction. The measurement error of the distance between the two vehicles is less than abo ut $10 \%$ as indi cated in the calibration experiment (5).

A total of 3061 data cas es including the near-crashes $(\mathrm{N}=3010)$ and crashes $(\mathrm{N}=51)$, was collected in the one-year field test. Figure 1(a) shows the distribution of near-crashes. Rear-end conflict data are the most numerous, which accounts for 39\% of the total. In the 51 accidents recorded, vehicle to cyclist accidents were the most numerous $(\mathrm{N}=18)$, which accounts for $35 \%$ of all accidents; rear-end accidents were second most common $(\mathrm{N}=17)$, which accounts for $33 \%$ of all accidents.

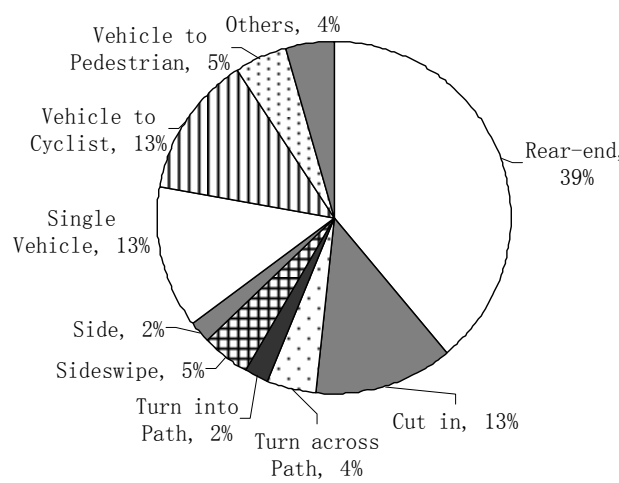

a) Near-crashes

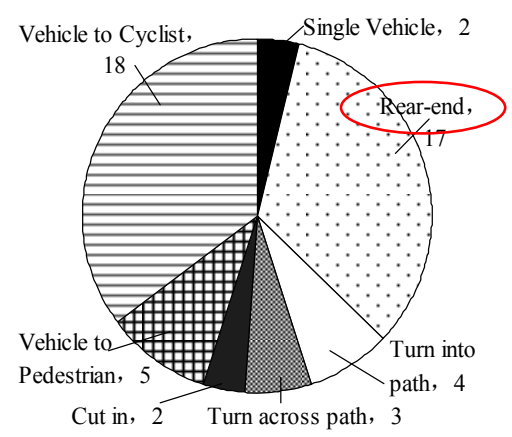

b) Crashes

Fig.1 Distribution of near-crashes and crashes recorded by VDR
The above data shows that, the number of near-crashes data recorded by VDR is 60 times than that of crashes data. If we can use relevant information from near-crashes effectively and grasp the characteristics of drivers' behavior and the impact factors accurately, that could ex ert a far-r eaching influence on understanding the mechanism of traffic accidents. However, nearcrashes and crashes were not the same and it is not confirmed as to what the relationship between near-crashes and crashes is.

Figure 2 shows the distribution of the comparison in accident types and occurrence time between near-crashes data and traffic accidents in Beijing in 2001 and 2002. When near-crash data in the field test is compared with data from traffic accidents in Beijing in 2001 and 2002, the distribution is v ery similar in both accident types and occurrence time.

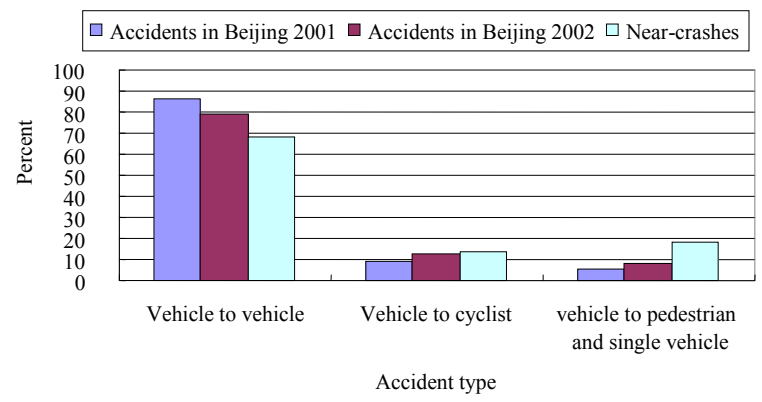

(a) Accidents type distribution

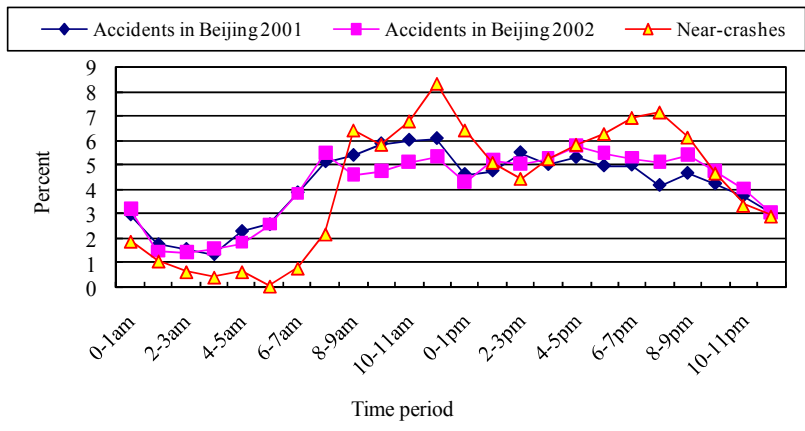

b) Time distribution

Fig.2 Comparison of near-crashes in this study and policereported crashes in Beijing 2001 \& 2002

Figure 3 shows the distribution of the results of the comparison in traffic accident types between $n$ ear-crashes data and crashes data in field test and we can see that the accident rate in vehicle to cyclist is higher. VDRs recorded a lot of emergency situations at intersections during red lights. Vehicle to vehicle near-crashes are further classified into several types, such as rearend, side scrape, cut-in, turn across path, turn into $\mathrm{p}$ ath and straight across path. The distribution of the results of crash and near-crash data is shown in Figure 4, which reveals that the distribution of rear-end near-crash types were quite similar in proportion. 


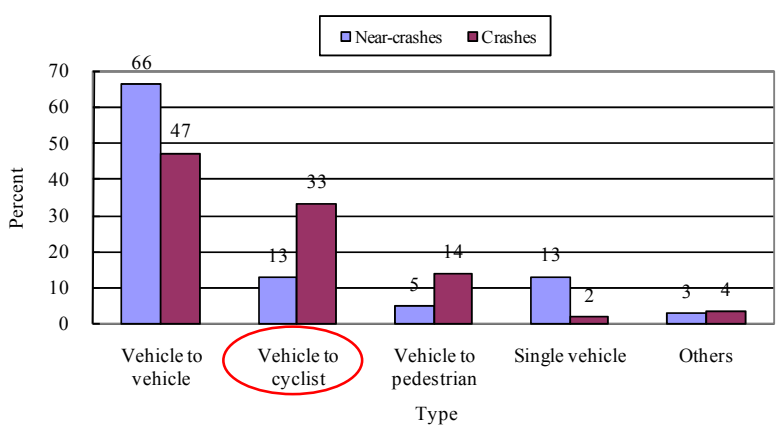

Fig.3 Comparison of accident types for near-crashes and crashes recorded by VDR

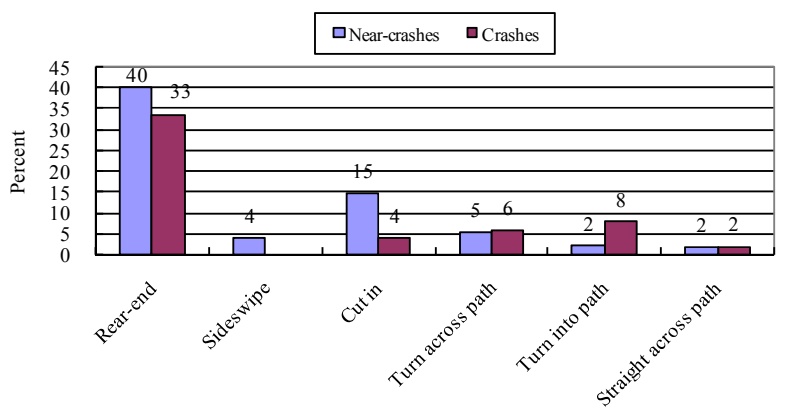

Type in vehicle to vehicle

Fig.4 Comparison of near-crashes types for near-crashes and crashes shown in Fig.3

Based on the above results, we can see that the near-crashes data and crashes data were qu ite similar. To a great ext ent, the characteristics of crash data can be described through the analysis of near-crash data. Figure 4 shows that the proportion of rear-end near-crashes is the high est, which accounts for $39 \%$ of all nearcrash data. The characteristics of avoid ance maneuvers an $d$ evaluation methods of near-cr ash data in rear-end near-crashes follows.

\section{Analysis of braking behaviors}

\subsection{Evaluation indices for driver braking operations}

For rear-end near-crashes, drivers generally use braking, steering, or co mbination of braking and steering to avoid accidents. The survey results show that the number of drivers who performed brake operation to avoid accidents accounted for about $95 \%$ of all drivers, the number of drivers who performed steering operation to avoid accidents only accounted for about $5 \%$ of all drivers. This is because near-crashes in the f ield test occurred mainly in low-speed and shorter following distance situations. Therefore, this study mainly analyzes the characteristic of driver brake operation.

Research into driver behavior and driver operation is usually performed by using indices as follows: using brake reaction time and brake operation speed to evaluate driver brake reaction speed; using average deceleration to evaluate the effects of driver brake operation; using maximum deceleration to evaluate driver braking ability. Brake reaction time (BRT) is defined as the time between braking onset of LV and braking onset of SV; driv er brake operation speed is evaluated by the Jerk (the rate of change of acceleration with respect to time) and the average deceleration is defined as the average value of deceleration of the vehicle from brake onset of S V till the shortest distance between SV and LV, that is, till the time when the relative velocity between the two vehicles becomes equal to zero.

\subsection{Characteristics of Driver Braking Operations}

Figure 5 indicates the changes in deceleration and velocity at different time when near-cr ashes occurred. Time $0(0 \mathrm{~s})$ is def ined as the time when the distance between the SV and LV is the shortest. As shown in Figure 5(a), there is not significant change in $\mathrm{SV}$ deceleration prior to $-2 \mathrm{~s}$ and there are great changes in $\mathrm{SV}$ deceleration after $-2 \mathrm{~s}$, in addition, the change from $-2 \mathrm{~s}$ to $-1 \mathrm{~s}$ is more rapid than those from $-1 \mathrm{~s}$ to $0 \mathrm{~s}$, which i llustrates that most of drivers perform braking op eration within the 2 s rang e before the most dangerous situation. As shown in Figure 5(b), there are almost no changes in SV velocity prior to $-2 \mathrm{~s}$ and as SV velocity starts to decrease, the rate from $-2 \mathrm{~s}$ to $-1 \mathrm{~s}$ is hig her than the rate from $-1 \mathrm{~s}$ to $0 \mathrm{~s}$.

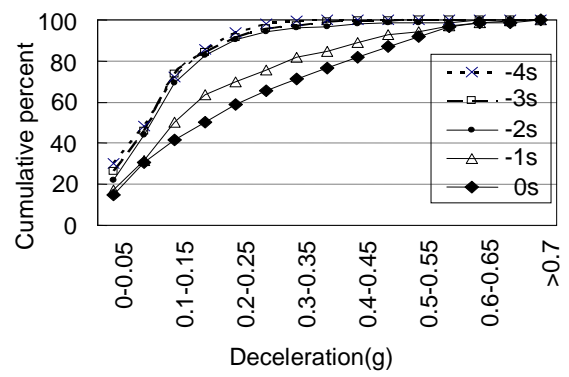

(a) Deceleration

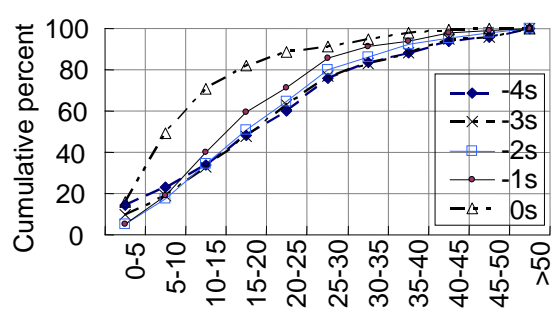

Travel Speed $(\mathrm{km} / \mathrm{h})$

(b) Velocity

Fig. 5 Variety of vehicle states at different time during nearcrashes $(\mathrm{N}=349)$

\subsection{Driving Behaviors of Conflict-prone Drivers}

Because of dri ver psychology, driver personality, driving skills etc, there is a certain type of person who suffers a greater number of near-crashes than normal. In this study, these drivers were called conflict-prone driver (CPD). I t has become widely accepted that driving behaviors of the CPD might bring about greater risk to traffic safety. A total of 2959 near-crashes data were collected, which include d personal max imum (365 nearcrashes) and personal minimum (8 near-crashes), the mean value 
of data $\mathrm{w}$ as 59. Accord ing to th e statistics of near- crashes occurrences, 50 drivers could $\mathrm{b}$ e divided into three groups, the Conflict-prone group was defined as the drivers who suffered a greater number of conf licts than normal, which accounted $f$ or $30 \%$ of all near-crashes (365-60 near-crashes per driver, 15 drivers). The Conflict-infrequent group (CID) w as defined as the drivers who suffered fewer number of near-crashes than normal, which accounted for $30 \%$ of all near-crashes (40-8 near-crashes per driver, 15 drivers). Behaviors characteristic of the CPD were analyzed in a comparison of driving behaviors of the two groups drivers.

Figure 6 indicates the comparison results of braking characteristics between the CPD and the CID. As it can be seen from Figure 6, there is a significant difference in maximum braking deceleration $(\mathrm{F}=4.55, \mathrm{p}<0.05)$ and average brak ing deceleration $(\mathrm{F}=4.42, \mathrm{p}<0.05)$ between the CPD and the CID, nevertheless, there is not a significant difference for BRT and Jerk between the CPD and the CID, which indicated that the CPD applied a relatively large braking force in the braking process.

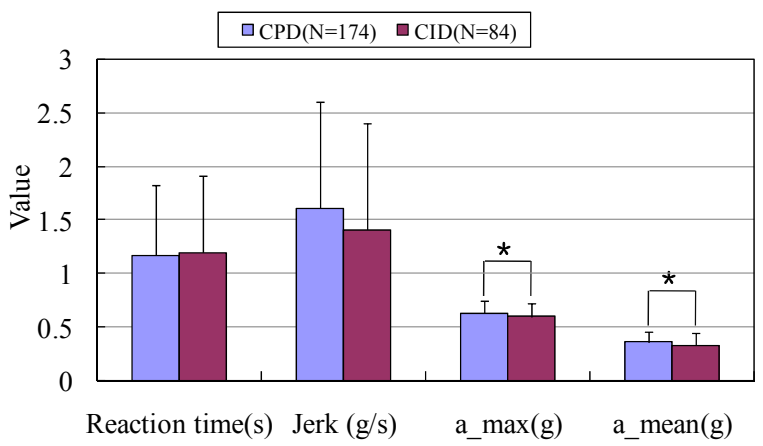

Fig.6 Characteristics of braking operations of the CPD and the CID

Figure 7 shows the vehicle statu ses at the onset of LV and SV braking between the CPD and the CID. As can be seen from Figure 7, the follow distance for the CPD is sho rter than that for the CIP ( $F=6.66, p<0.05)$, and the relative velocity for the CPD is also less than that for the CIP at LV braking onset ( $F=5.43, p$ $<0.05)$ and SV braking onset $(\mathrm{F}=6.9, \mathrm{p}<0.05)$, but there is no difference in velocity, which is middle-low. In addition, there is not a signif icant difference in vehicle statuses at differ ent time except when the relative velocity increased at the SV braking.

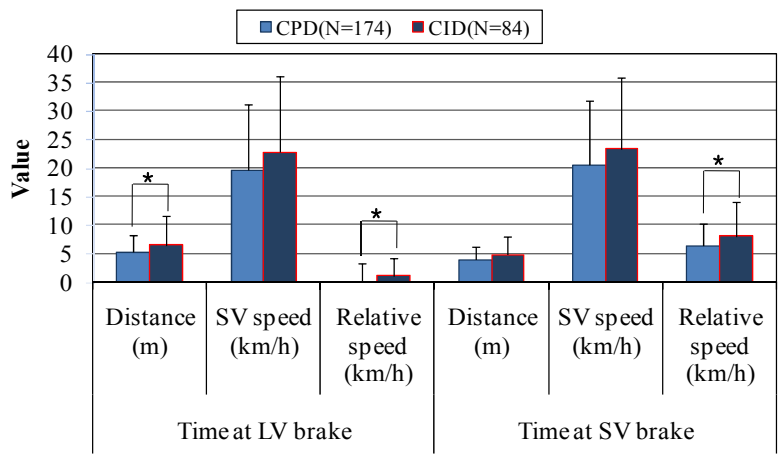

Fig.7 Vehicle statuses at the onset of LV and SV braking for the CPD and the CID
Driving behaviors of $\mathrm{LV}$, which can have a significan $\mathrm{t}$ impact on drivers' judgment and oper ation when following a vehicle, consequently, have a si gnificant impact on drivers' avoidance maneuvers as well when near-crashes occurred (i.e. when someone brakes suddenly). In this study, the pre-event behaviors of LV were divided into accelerating and moving at a constant speed; the changes of the SV drivers operation were comparatively analyzed (Figure 8). As can be seen from Figure 8, both the CPD and the CID quickly followed and accelerated (72\%) or held a constant speed (28\%) in the same pro portion when LV accelerated. However, when the LV held a constant speed, the ratio of the CPD who accelerated (40\%) was significantly greater than that of the CID (20\%), which shows that, in traffic jams most drivers might choose to accelerate, and thus reduce the following distance to avoid other vehicles cutting-in. When the LV held a constant speed, the number of the CPD who chose to accelerate to shorten the following distance was greater than the CID, which coincides with the results that the following distance of the CPD was shorter (Fig ure 7). An analysis of the results of th e abovementioned studies shows that the CPDs have aggressive driving characteristic because of short following distance, larger deceleration and frequently accelerating to reduce following distance, etc.

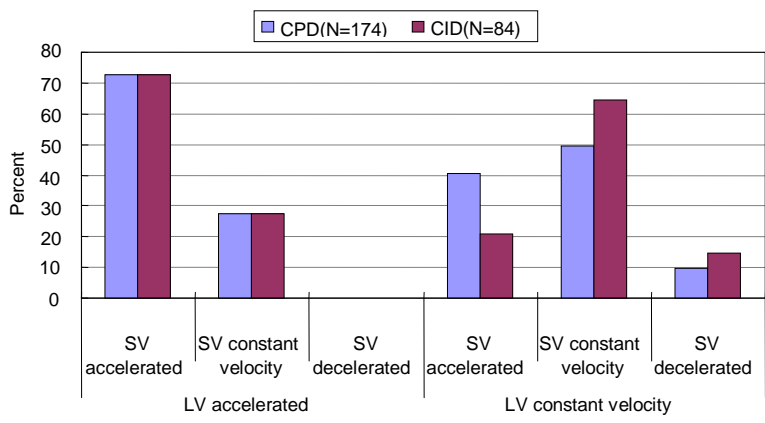

Fig.8 Driving operations of LV accelerating and running with a constant velocity for the CPD and the CID

\section{Evaluation of avoidance maneuvers effects}

\subsection{Evaluation method}

During vehicle following, the starting point(time zero poin $t$ ) was defined as the time of braking onset of the LV. at LV braking onset, the following distance, the velocity of the LV, the velocity of the $\mathrm{SV}$, the relative velocity, the deceleration of the LV and the deceleration of the SV are $D_{0}, V_{L}, V_{S}, \Delta V=V_{S}-V_{L}, a_{L}$ and $a_{S}$, respectively. Meanwhile, the moving state of the LV prior to crashing can be divided into stopped and mov ing, the vehicle status parameters of both the LV and the SV are shown in Figure 9. 


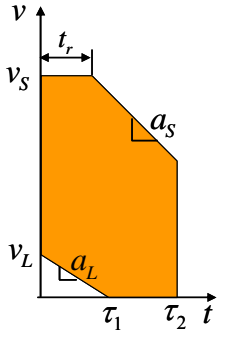

(a)

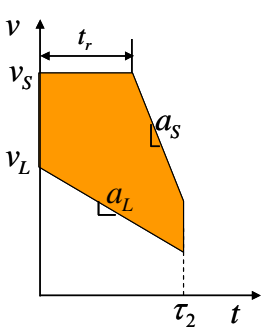

(b)
Fig.9 Vehicle state parameters of the LV and the SV during a near-crash

(1) LV stopped prior to crashing

At the time zero point, the LV applies the brake at the rate of $a_{L}$ and stops at the time of $\tau_{1}$, and then the SV starts to brake after reaction time $t_{r}$, and collides with the stopped LV at the time of $\tau_{2}$ (Figure 9(a)). The relative distance was calculated by the following formula:

$$
D=D_{0}-V_{S} \cdot t_{r}-\frac{V_{S}^{2}-V_{S t}^{2}}{2 \cdot a_{S}}+\frac{\left(V_{S}-\Delta V\right)^{2}}{2 \cdot a_{L}}
$$

Considering both the following distance and the relative velocity being zero as the critic al condition to avoid collision, therefore, the relationship between the deceleration $a_{s}$ and the reaction time $t_{r}$ of the $\mathrm{SV}$ can be obtained, as shown in formula (2):

$$
a_{S}=\frac{a_{L} \cdot V_{S}^{2}}{2 \cdot a_{L} \cdot D_{0}-2 \cdot a_{L} \cdot V_{S} \cdot t_{r}+\left(V_{S}-\Delta V\right)^{2}}
$$

\section{(2) LV moving prior to crashing}

The LV applies the brake at the rate of $a_{L}$ and the SV starts to brake after reaction time $t_{r}$, collides with the moving LV at the time of $\tau_{2}$ (Figure 9(b)). The relative distance was calculated by the following formula:

$$
D(t)=D_{0}+V_{L} \cdot t-\frac{1}{2} \cdot a_{L} \cdot t^{2}-V_{S} \cdot t+\frac{1}{2} \cdot a_{S} \cdot\left(t-t_{r}\right)^{2}
$$

While the discriminant is greater or equal to zero $(\Delta \geq 0$, indicates the $\mathrm{LV}$ is moving before the collision and the relative distance $\geq 0$ ), the formula (3) has the solution. And while $\Delta=0$, the critical condition can be obtained, as shown in formula (4):

$$
a_{S}=-\frac{\Delta V^{2}+2 \cdot a_{L} \cdot D_{0}}{a_{L} \cdot t_{r}^{2}+2 \cdot \Delta V \cdot t_{r}-2 \cdot D_{0}}
$$

\subsection{Case Study}

Figure 10 shows a rear-end accident that occurred on an arterial highway in Beijing recorded by VDR. Figure 10(a) shows a part of th e video image dur ing the course of the accident and Figure 10(b) s hows the brak ing operation and the status parameters of the SV. The time zero point (0s) in Figure 10 was set for the time of collision. The accident scenario is shown as follows: the SV followed the LV at the speed of approximately $50 \mathrm{~km} / \mathrm{h}$, changed to the right lane and try to overtake at $-6.0 \mathrm{~s}$, at that moment, another car was cutting in from the right lane in front of the SV; therefore, the SV had to stop the lane change and return to the original lane $(-4.8 \mathrm{~s})$, where the LV performed a sudden brake with the deceleration of approximately $0.25 \mathrm{~g}$ and stopped $(-3.5 \mathrm{~s})$, after a $1.7 \mathrm{~s}$ delay, the SV braked at $0.4 \mathrm{~g}$. Due to the short following distance $(5.7 \mathrm{~m})$, the SV collided with the LV at $23 \mathrm{~km} / \mathrm{h}(0 \mathrm{~s})$ finally. The LV had stopped prior to crashing, therefore the critical condition, which is the collision line in Figure 11, can be calculated by formula (2) in part 4.1.

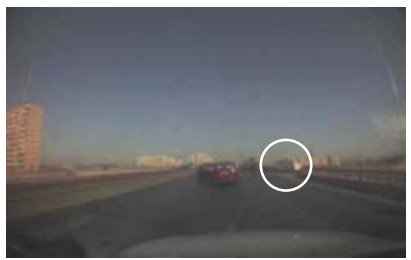

(a) Another car cutting-in from the right lane at $-4.8 \mathrm{~s}$

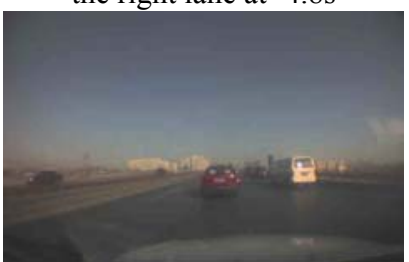

(c) SV braking onset at-1.8s

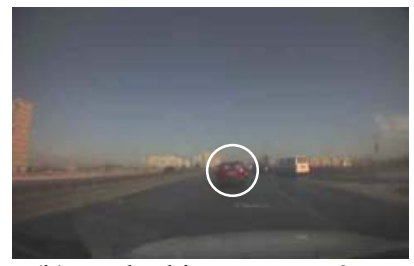

(b) LV braking onset at-3.5s

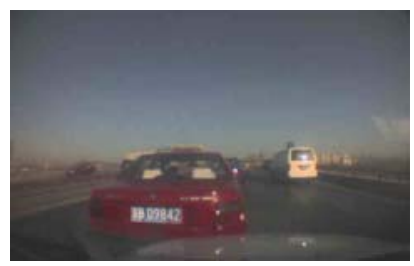

(d) Collision occurrence at $0 \mathrm{~s}$ (a) Rear-end accident scenarios

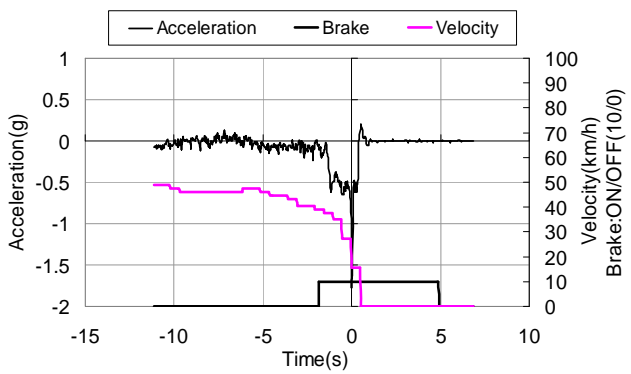

(b) Data of SV in rear-end accident

Fig.10 A rear-end accident occurred on an arterial road at dusk recorded by VDR

As shown in Figure11, the ho rizontal axis represents the braking level (average deceleration) of the SV a nd the longitudinal axis represents the braking reaction time of the SV. The bold line in the figure is the collision line, which was calculated according to the above-mentioned conditions. Above the collision line is the $\mathrm{c}$ rash region and below that is the nearcrash region; the fine line represents the contour line of the collision speed, and the number for each contour line is the collision speed. At any casual point, the coordinate values (braking levels and braking reaction time), represents the braking operation to avoid the crash, and whether collision occurs or not, which is determined by whether the casual point is in the crash region or near-crash region. In addition, the collision speed when collision occurs can be distinguished according to the relative 
location relationship between that point and the contour line of each collision speed. Point A $(0.4 \mathrm{~g}, 1.7 \mathrm{~s})$, which is between the contour line 20 and 25 is consis tent with the collision speed of $23 \mathrm{~km} / \mathrm{h}$ recorded by VDR, sho ws the real $\mathrm{b}$ raking deceleration and reaction time of the driver during the accident as shown in Figure10.

Both the braking reaction time and the braking level are the two key parameters that can determine the effects of collision avoidance, and what kind of methods may help to avoid the crash can be directly distinguished under the current circumstances by using Figure 11. With respect to the rear-end accident shown in Figure10 for instance, if the SV applies the brake $0.3 \mathrm{~s}$ in advance (point $\mathrm{B}$ ), or the $\mathrm{SV}$ deceleration is $0.55 \mathrm{~g}$ (point $\mathrm{C}$ ), the accident can be avoided. Figure 12 shows the simulation results of the changes between the ve locity and the $\mathrm{r}$ elative distance in the avoidance method, such as A, B and C, and as can be seen, the collision can be avoided in the second and third methods.

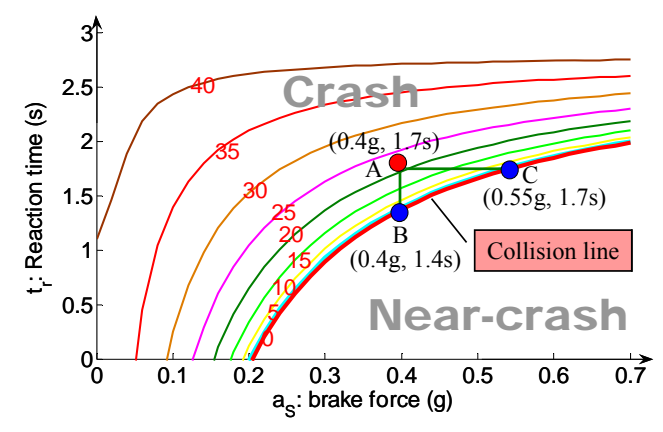

Fig.11 Critical condition of the case shown in Figure11 regarding accident avoidance

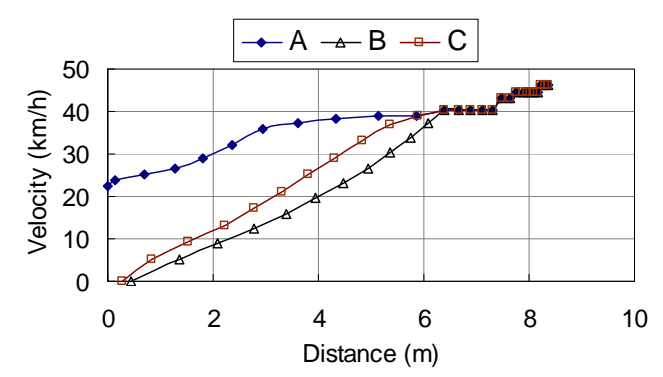

Fig.12 Variety of following distance under different avoidance methods

For accidents due to de layed reaction, insufficient braking, etc, the Driver Assistance System (DAS), such as the Brake Assist System (BA), the Forward Collision Warning System (FCWS) and the Pre-crash Safety System (PCS), etc. can be used to help the drivers improve the response speed and increase the braking level to avoid the crash. The methods shown in Figure 11 can be applied in the following areas. In the research and development phase of system design, the effective of var ious DAS could be predicted according to the driver's response and op erating characteristics in a variety of typical conditions. The method of assistance could be s elected, for example, whether to us e the FCWS to help the driver improve the response speed or to use the BA to help the driver increase the braking level, then the guidance for optimization of the DAS could be $g$ iven. In actual use, the effects on drivers' operations can be assessed in real-time, which determine when and in what ways the drivers should be assisted . For example, for the PCS, based on the current state of the vehicle and the operating behavior of the driver, the collision speed could be predicted by using the results and methods shown in Figure 11, and according to the pre-set safety goals, the timing and th e braking level of intervene in the PCS could be determined to achieve quantitative control of the PCS.

\subsection{Analysis of accidents avoidance effects}

The drivers performed the braking operation obviously in 6 of the rear-end accidents. According to the methods shown in Figure 11, how much more braking reaction time and brakin $\mathrm{g}$ level was needed to avoid the collision are shown in Table 1. As can be seen, if the SV applies the brake $0.4 \mathrm{~s}$ in advance, all the accidents could be avoided and if the braking force of $0.2 \mathrm{~g}$ is increased, 4 accidents could be avoided, and another 2 accidents could not be avoided through increasing the braking level.

Table 1 Analysis of effects of accident avoidance

\begin{tabular}{|c|c|c|c|c|}
\hline ID & $\begin{array}{c}\text { Reaction } \\
\text { Time (s) }\end{array}$ & $\begin{array}{c}\text { Brake } \\
\text { timing } \\
\text { advanced (s) }\end{array}$ & $\begin{array}{c}\text { Braking } \\
\text { force(g) }\end{array}$ & $\begin{array}{c}\text { Braking force } \\
\text { increased (g) }\end{array}$ \\
\hline A1 & 1.2 & 0.3 & 0.5 & Impossible \\
\hline A2 & 1.33 & 0.3 & 0.35 & 0.2 \\
\hline A3 & 0.77 & 0.4 & 0.5 & 0.2 \\
\hline A4 & 1.97 & 0.1 & 0.55 & 0.05 \\
\hline A5 & 0.6 & 0.2 & 0.45 & 0.1 \\
\hline A6 & 0.87 & 0.3 & 0.55 & Impossible \\
\hline
\end{tabular}

100 rear-end near-crash cases were extracted from 1217 rearend near-crash cases. The time delay leading to the accidents was calculated for every near-crash case by using the methods shown in Figure 11; Figure 13 shows the statistics for the 100 rear-end near-crash cases analysis. As can be seen from Figure 13, if the $\mathrm{SV}$ is delay ed $0.2 \mathrm{~s}$, about $18 \%$ of near-crashes would beco me crashes; if the SV is delay ed $1.0 \mathrm{~s}$, about $59 \%$ of near-crashes would become crashes.

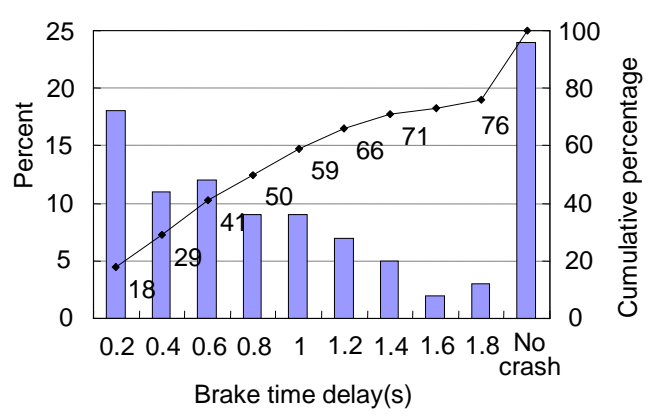

Fig.13 Accidents rates due to braking time delay for the 100 nearcrashes recorded by VDR

Similarly, how much deceleration leading to the accidents was also calculated for every near-crash case, Figure 14 shows the statistics of accidents rates due to deceleration for the 100 rearend near-crashes cases analysis. As can be seen from Figure 14, if the SV applies the brake with a deceleration rate of $0.1 \mathrm{~g}$, about 
$33 \%$ of near-crashes would become crashes; with a deceleration rate of $0.2 \mathrm{~g}$, about the $64 \%$ of near-crashes would become crashes.

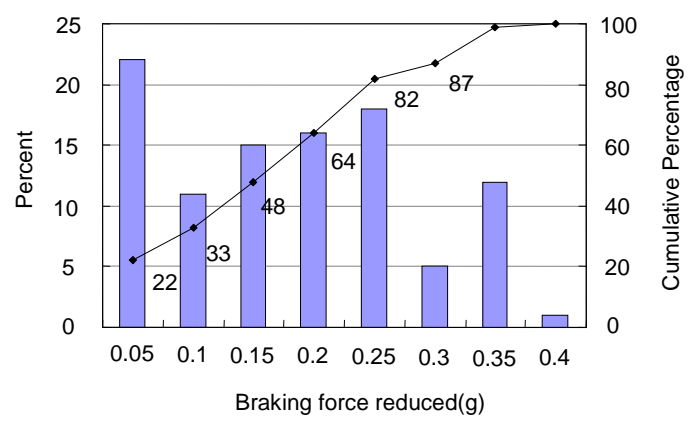

Fig.14 Accidents rates due to deceleration reduced for the 100 near-crashes recorded by VDR

For every near-crash, both the delay of braking and the deceleration rate of the vehicle leading to the accidents can be calculated simultaneously. Figure 15 shows the st atistics of accidents rates due to the changes of deceler ation and brake timing for the 100 rear-end near-crash cases. As can be seen from Figure16, the horizontal axis represents the delay of braking, the longitudinal axis represents the decrease in braking, and the area with the $\mathrm{s}$ ame texture represents the $\mathrm{s}$ ame accident rate. For example, point $\mathrm{O}(0.1 \mathrm{~s}, 0.2 \mathrm{~g})$ in the figure shows that, if the $\mathrm{SV}$ is delayed $0.1 \mathrm{~s}$ and the SV applies the brake with a deceleration rate of $0.2 \mathrm{~g}$, about $70 \%$ near-crashes would become crashes. Also, as can be seen, both the point $(0.6 \mathrm{~s}, 0.1 \mathrm{~g})$ and the point $(0.95 \mathrm{~s}, 0.0 \mathrm{~g})$ have the same accident rate as $\mathrm{O}(0.1 \mathrm{~s}, 0.2 \mathrm{~g})$. Using the results shown in Figure 15 and considering the perfor mance of $v$ arious DAS and $r$ esponse characteristics of drivers to the DAS, the effects of various DAS in operation could be evaluated quantitatively.

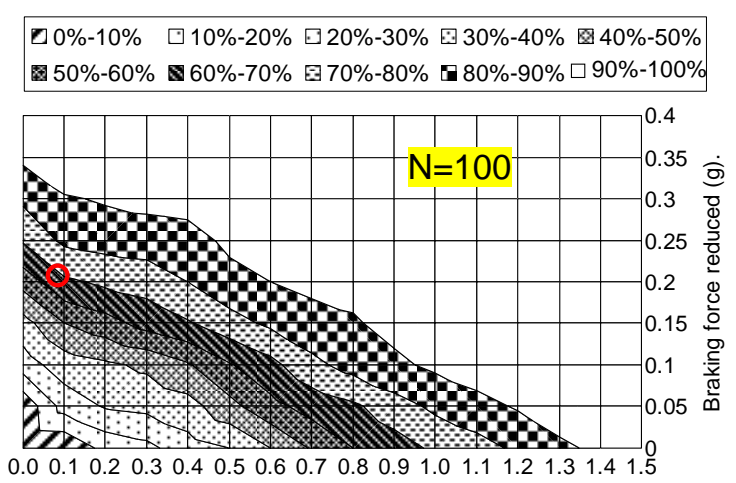

Brake timing delay (s)

Fig. 15 Accidents rates due to the changes in deceleration and the brake timing for the 100 near-crashes recorded by VDR

Figure 15 shows the statistics of accidents rates due to the changes in deceleration and the brake timing for the 100 rear-end near-crash cases. As a r esult, the sample size will have a greater impact on the results. Figure16 shows the influence on the stability of results shown in Fig. 15 due to the variety of sample numbers. As can be seen from Figure 16, the statistic results in this study were compared among the four size conditions, such as $30,50,70$, and 100, respectively. Firstly, 30 rear-end near crashes cases from different drivers were selected randomly, and then, on the basis of 30 rear-end near crashes cases selected, the number of rear-end near crashes was enlarged from 30 to 50 or above fro $\mathrm{m}$ different drivers. Meanwhile, when the sample size changes from 30 to 50 , or to 70 , the distribution region of accident rates change greatly. In addition, as the sample size is increased to 70 , the distribution region of accid ent rates tends to stay steady, which indicates that the results acquired by using 100 near-crash cases have some reference value as shown in Figure15.
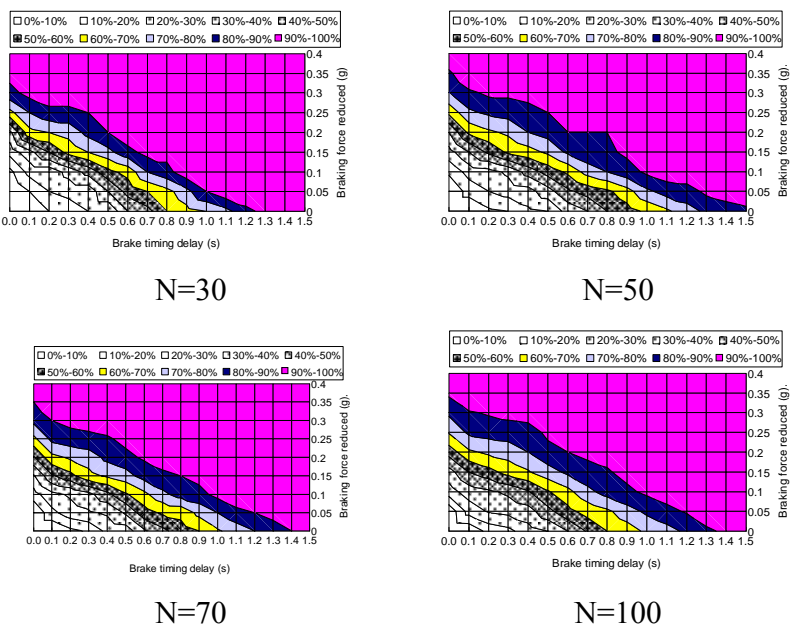

Fig.16 Influence on the stability of results shown in Fig. 15 due to the variety of sample numbers

\section{Conclusion}

In this study, a large-scale of one-year field test in Beijing was conducted using 50 taxis equipped with VDRs, and a large volume of natur alistic driving data including crashes and nearcrashes was collected; moreover, drivers' braking operations in emergency situations were studied. The results are as follows:

By contrasting the near-crashes with crashes recorded by the VDR regarding the traffic accidents in Beijing, there existed great similarities between them, regardless of type or time of occurrence. Thereby, the characteristics and mechanism of crashes can be described through the study of near-crashes.

Most evasive maneuvers for drivers were within $2 \mathrm{~s}$ before the most dangerous state. The studies involved the braking operations of the drivers in $r$ ear-end near-crashes. There is no significant difference between the conflict-prone drivers (CPD) and other drivers in $\mathrm{r}$ eaction time and br aking speed, but th $\mathrm{e}$ conflict-prone drivers (CPD) showed sev eral behavioral characteristics, such as sh ort following distance, larger deceleration and frequent acceleration.

An evaluation method was proposed to quantitatively evaluate the effect of avoidance maneuvers, which could be used to accurately predict the probability of accidents occurring and the collision speeds under various braking conditions. Th e relationships between accident rates and the ch anges of 
characteristics of braking op erations were analyzed using the near-crash data recorded by the VDR, which could provide support to the design and improvement of safety assistant systems (e.g., PCS).

\section{Acknowledgements}

This study belongs to the cooperative research with Toyota Motor Corporation of Jap an and the authors would like to thank Toyota Motor Corporation for the financial support provided and assistance in this research and this study was also supported b y the Fundamental Research Funds for the Central Universities.

\section{References}

(1) Dingus, T. A., Klauer, S. G., Neale, V. L., Petersen, A., Lee, S. E., Sudweeks, J., et al. The 100-car naturalistic driving study, Phase II - r esults of the 100-car field experiment. (Report No. DOT HS 810 593).Washington, DC: National Highway Traffic Safety Administration, (2006).

(2) Klauer, S. G., Dingus, T. A., Neale, V. L., Sudweeks, J .D., and Ramsey, D. J.. The Impact on Driver Inattention on Near Crash/Crash Risk: An Anal ysis Using the 100 Car Natur alistic Driving Study Data. (Report No. DOT HS 810 594). Washington, DC: National Highway Traffic Safety Administration, (2006).

(3) N. Kubo, S. Kitajima, T. Katayama, et al. "Analysis of rearend collision near-miss and its occurrence by drive data recorder", Proceedings. JSAE Annual Co ngress, no. 12 6-06, pp. 7-10, (2006).

(4) Nishimoto, T., Yoshimoto, K., Nishida, H., et al. Development and Validation of a Drive-Recorder for Automobile Accidents," JSAE Paper 20005174, (2000).

(5) Qingfeng Lin, Bo Cheng, Ju nke Lai, et al. A new method for analysis of tr affic conflict by using $\mathrm{v}$ ideo drive $\mathrm{r}$ ecorders. Proceedings ITS Conference. Beijing: ITS Conference, (2007).

(6) Qingfeng Lin, Ruijia Feng, Bo Cheng, et al. Analysis of Causes of Rear-end Conflicts Using Naturalistic Driving Data Collected by Video Drive Recorders. SAE A nnual Congress. Proceedings SAE Annual Con gress. New York: SAE Annua 1 Congress, (2008). 